J. Lake Sci.(湖泊科学), 2009, 21(3): 369-374

http://www.jlakes.org. E-mail: jlakes@niglas.ac.cn

(c)2009 by Journal of Lake Sciences

\title{
与微囊藻胞外多糖降解相关的微生物菌群分析*
}

\author{
蔡元锋 ${ }^{1,2}$, 施丽梅 ${ }^{1}$, 李朋富 ${ }^{1,2^{* *}}$, 邢 鹏 $^{2}$, 于 $\quad$ 洋 $^{2}$, 孔繁翔 ${ }^{2}$ \\ (1: 南京大学生命科学学院, 南京 210093) \\ (2: 中国科学院南京地理与湖泊研究所湖泊与环境国家重点实验室, 南京 210008)
}

摘 要: 从铜绿微囊藻培养液中提取胞外多糖, 化学分析显示其为含 $8.3 \%$ 蛋白质的酸性杂多糖. 以胞外多糖为碳源接种自然 水体的菌群进行富集培养, 分析胞外多糖的微生物降解过程及降解菌的组成. 结果表明, 接种太湖水华期微生物富集菌群后, 多糖立即开始被降解, 大约在 $18 \mathrm{~d}$ 后多糖降解过程显著减慢, 37d 后仍有一部分多糖未能被降解. 这些结果说明在自然界中微 囊藻胞外多糖是可以被微生物降解的. 比较不同水体的菌群对多糖的降解能力后显示, 降解菌群只存在于微囊藻水华暴发的 阶段. 变性梯度凝胶电泳(DGGE)结果显示在整个降解过程中, 降解菌群的组成未发生显著变化. 对 DGGE 条带中的 DNA 片 段进行序列分析和系统发育分析表明降解菌群包含以下几类：鞘氨醇单胞菌属(Sphingomonas)3 个, 褐螺菌属 (Phaeospirillum) 1 个, 假单胞菌属(Pseudomonas) 1 个, 红环菌科(Rhodocyclaceae) 1 个, Hylemonella(无译名)1 个, 分枝杆菌属 (Mycobacterium) 1 个.

关键词: 微囊藻; 胞外多糖; 降解; 变性梯度凝胶电泳; 菌群

\section{Composition of bacterial community related to degrading the exopolysaccharide from the cyanobacterium Microcystis aeruginosa}

CAI Yuanfeng ${ }^{1,2}$, SHI Limei ${ }^{1}$, LI Pengfu ${ }^{1,2}$, XING Peng ${ }^{2}$, YU Yang ${ }^{2}$ \& KONG Fanxiang ${ }^{2}$

(1: School of Life Sciences, Nanjing University, Nanjing 210093, P.R.China)

(2: State Key Laboratory of Lake Science and Environment, Nanjing Institute of Geography and Limnology, Chinese Academy of Sciences, Nanjing 210008, P.R.China)

Abstract: The acidic heteropolysaccharide released by a water-bloom cyanobacterium Microcystis aeruginosa FACHB-912, which contained $8.3 \%$ protein, was used as the carbon source in stable enrichment cultures inoculated with natural aquatic microbial communities. The process of microbial breakdown of the exopolysaccharide and composition of the microbial community related to degrading exopolysaccharide were investigated. After inoculation of aquatic microbial community from Lake Taihu, enrichment cultures were obtained. In the enrichment culture, the exopolysaccharide was degraded immediately. The breakdown of the exopolysaccharide slowed markedly after about 18d, left a nondegraded fraction after 37d. The above data, together with comparison experiments on degradation capability of bacterial communities from various aquatic environments, indicated that the exopolysaccharide from M. aeruginosa FACHB-912 could indeed be degraded by microorganisms coexisting only with the water bloom in nature. Denaturing gradient gel electrophoresis (DGGE) analysis showed composition of the microbial community did not change significantly during exopolysaccharide degradation. Phylogenetic analysis on DNA fragments, which were excised from DGGE gels, placed three degraders in Sphingomonas, one in Phaeospirillum, one in Pseudomonas, one in Rhodocyclaceae, one in Hylemonella, and one in Mycobacterium.

Keywords: Microcystis aeruginosa; exopolysaccharide; microbial degradation; DGGE; bacterial community

* 江苏省自然科学基金(BK2007150)、国家重点基础研究发展计划(2008CB418004)和中国科学院王宽诚博士后工作奖励基 金联合资助. 2008-08-10 收稿; 2008-09-18 收修改稿. 蔡元锋, 男, 1983 年生, 硕士研究生; E-mail: wcyfhgl@126.com.

** 通讯作者; E-mail: pengfuli@nju.edu.cn. 
由于水体富营养化而导致的蓝藻水华在世界范围内频繁发生, 微囊藻(Microcystis spp.)是形成蓝藻 水华的主要种类之一，在我国尤为常见．微囊藻胞外多糖为酸性杂多糖，能够富集金属离子, 影响水体 中金属离子的溶解、沉积、生物利用和循环; 胞外多糖将微囊藻细胞包裹在一起形成群体，可以阻止一 些浮游动物的吞食, 保持其在水体中的生存竞争优势, 并可使水华持续较长的时间; 一部分微囊藻胞外 多糖溶解到周围水体中, 不仅影响到水体中多糖的浓度, 还可以与微生物和碎屑粘结形成有机颗粒 (organic aggregates) $^{[1-2]}$, 使水体浑浊度增加.

在英国的 1 个湖泊中调查发现, 水华发生时包裹着微囊藻群体的胞外多糖可以占水体变温层 (epilimnion)体积的 $0.007 \%$, 在水华高峰期可以占到 $0.06 \%{ }^{[3]}$. 作为水体中异养微生物生长的重要营养源, 藻类胞外多糖影响水体中微生物的丰度和多样性 ${ }^{[4]}$. 水华暴发过程中湖泊水体中长时间地存在大量的 微囊藻胞外多糖, 微囊藻胞外多糖的微生物降解过程是湖泊碳循环的重要环节. 本文旨在研究微囊藻 胞外多糖的微生物降解过程和降解菌群的组成, 为探讨蓝藻水华的暴发规律及蓝藻水华的防治提供新 的基础信息.

\section{1 材料与方法}

\section{1 微囊藻的培养及其胞外多糖的提取}

铜绿微囊藻(Microcystis aeruginosa FACHB-912)购自中国科学院水生生物研究所, 该藻株由雷腊梅 在 1997 年分离自太湖. 2L 藻液培养在 3L 雉形瓶中, 培养基为 BG11, 培养过程中通人过滤空气, 光强 $2000 \mathrm{~lx}$ ，光暗比 $12 \mathrm{~h}: 12 \mathrm{~h}$ ，温度 $25^{\circ} \mathrm{C}$. 培养 $30 \mathrm{~d}$ 的藻液 $6000 \mathrm{r} / \mathrm{min}$ 离心 $10 \mathrm{~min}$ ，收集上清液，上清液依次用 $0.8 \mu \mathrm{m} 、 0.45 \mu \mathrm{m}$ 和 $0.22 \mu \mathrm{m}$ 的微孔滤膜抽滤，滤液用蒸馏水透析(透析袋截留分子量 $7000 \mathrm{Dalton}) 72 \mathrm{~h}, 45^{\circ} \mathrm{C}$ 减压浓缩后, 部分作为铜绿微囊藻胞外多糖(MEPS)母液用于微生物降解和化学组成分析, 部分冻干用于 化学组成分析.

\subsection{MEPS 的化学组成分析}

1.2.1 中性单糖组成分析 称取冻干 MEPS 样品 $10 \mathrm{mg}$ 放人安鶕瓶中, 加人 $2 \mathrm{~mol} / \mathrm{L}$ 三氟乙酸 $1.5 \mathrm{ml}$, 充人氮 气, 烧融封口, 在 $120^{\circ} \mathrm{C}$ 下水解 $3 \mathrm{~h}$, 水解后的糖溶液用过滤空气吹干后, 按照李铁林等的方法进行糖腈衍 生化 ${ }^{[5]}$. 具体方法如下: 加人 $10 \mathrm{mg}$ 盐酸差胺和 $0.5 \mathrm{ml}$ 吡啶, $90^{\circ} \mathrm{C}$ 水浴 $30 \mathrm{~min}$ 并振荡, 取出后冷至室温, 加 人 $0.5 \mathrm{ml}$ 醋酸酐, 在 $90^{\circ} \mathrm{C}$ 下继续反应 $30 \mathrm{~min}$ 进行乙酰化, 反应完成后进行气相色谱分析. 单糖标准品处理 同上.

气相色谱条件：仪器为 HP-6890气相色谱仪，色谱柱为 $\mathrm{HP}-55 \%$ 苯甲基硅烷 $(30 \mathrm{~m} \times 0.25 \mathrm{~mm} \times 0.25 \mu \mathrm{m})$; 流量 $1.2 \mathrm{ml} / \mathrm{min}$; 进样温度 $260^{\circ} \mathrm{C}$; 程序升温: $146^{\circ} \mathrm{C}$ 保留 $2 \mathrm{~min}$, 从 $146^{\circ} \mathrm{C}$ 以 $2^{\circ} \mathrm{C} / \mathrm{min}$ 升温到 $210^{\circ} \mathrm{C}$, 从 $210^{\circ} \mathrm{C}$ 以 $30^{\circ} \mathrm{C} / \mathrm{min}$ 升温到 $280^{\circ} \mathrm{C}$; FID检测器, 检测温度 $300^{\circ} \mathrm{C}$, 载气为氮气; 进样量 $1 \mu \mathrm{l}$.

1.2.2 糖醛酸定性和定量分析 糖醛酸定性分析: MEPS 的水解方法同上, 干燥后的水解产物加人 1-苯基 -3-甲基-5-吡唑啉酮(PMP, $0.5 \mathrm{~mol} / \mathrm{L}$ 甲醇溶液) $20 \mu \mathrm{l}$ 和 $20 \mu \mathrm{l} \quad 0.3 \mathrm{~mol} / \mathrm{L}$ 氢氧化钠溶液, $70^{\circ} \mathrm{C}$ 水浴反应 $30 \mathrm{~min}$, 衍生产物加人 $30 \mu \mathrm{l} 0.3 \mathrm{~mol} / \mathrm{L}$ 盐酸溶液中和. 为了去除多余的试剂, 衍生产物使用氯仿萃取 2 次, 最终得 到的水相产物用于 HPLC 分析. 糖醛酸标准品处理同上.

HPLC 条件：色谱柱：ZORBAX SB-C18(150mm×4.6mm); 流动相: $0.1 \mathrm{~mol} / \mathrm{L}$ 磷酸盐缓冲液-乙腈 (V:V=83:17); 流速: $0.7 \mathrm{ml} / \mathrm{min}$; 柱温: $30^{\circ} \mathrm{C}$; 检测波长: $245 \mathrm{~nm}$.

糖醛酸定量分析采用咔唑-硫酸法分析 ${ }^{[6]}$. 于带塞试管中加人 $0.8 \mathrm{ml}$ MEPS 溶液, 然后向试管中分别 加人 $4 \mathrm{~mol} / \mathrm{L}$ 氨基磺酸和 $\mathrm{pH} 8.4$ 的嗍酸缓冲液各 $0.1 \mathrm{ml}$, 混匀后再加人 $96.4 \%$ 的浓硫酸 $5 \mathrm{ml}$, 于 $100^{\circ} \mathrm{C}$ 水浴 锅中水浴 $6.5 \mathrm{~min}$, 冷却后加 $0.2 \mathrm{ml} 0.2 \%$ 咔唑乙醇溶液混匀, $100^{\circ} \mathrm{C}$ 水浴锅中水浴 $10 \mathrm{~min}$, 冷却后于 $525 \mathrm{~nm}$ 测 OD 值，以葡萄糖醛酸为标准品绘制标准曲线，由标准曲线计算出糖醛酸含量.

1.2.3 蛋白质和金属元素含量分析 采用南京建成生物工程研究所的考马斯亮兰法蛋白测定试剂盒测定 蛋白含量. 用 ICPS-1000 II 型电感耦合等离子体发射光谱仪(日本岛津公司产品)分析金属元素的含量.

\subsection{MEPS 降解过程分析}

接种所用的微生物水样分别在 2007 年 8 月、12 月即微囊藻水华期和无水华期取自太湖梅梁湾, 8 月 
还在南京市浦口区一个鱼塘采集了微生物水样, 该鱼塘 10 多年无蓝藻水华发生, 所采样品作为无水华水 体水样. 2008 年 5 月再次在太湖梅梁湾采集了水华期微生物水样. 水样取回后立即用无菌 Whatman GF/F 膜过滤以除去原生动物、藻类和其他颗粒物质. $5 \mathrm{ml}$ 滤液接种到 $100 \mathrm{ml}$ 培养基(成分为: $50 \mathrm{ml}$ 含 BG11 培养 基各成分的浓度约 $230 \mathrm{mg} / \mathrm{L}$ 的 MEPS $+50 \mathrm{ml}$ 无菌太湖水)中, $25^{\circ} \mathrm{C}$ 条件下暗处进行富集培养, 每天定期摇动, 每 15-20d 后转接到 10 倍体积的新鲜培养基中. 将稳定的富集培养菌液接种于同样的培养基, 在相同的 培养条件下进行降解过程分析. 降解过程中总碳水化合物的含量变化分析：取 $1 \mathrm{ml}$ 培养液样品, $8000 \mathrm{r} / \mathrm{min}$ 离心 $15 \mathrm{~min}$, 上清用苯酚-硫酸法 ${ }^{[7]}$ 检测碳水化合物的量. 降解菌生物量的变化分析: 取 $1 \mathrm{ml}$ 菌 液用 $2 \%$ 甲醛固定, DAPI 染色后用苂光显微镜计数 ${ }^{[8]}$. 所有降解实验均重复 3 次.

\subsection{PCR-DGGE 分析降解菌组成}

在分析MEPS降解过程中定期取 $1 \mathrm{ml}$ 菌液, 离心后将细菌保存于 $-20^{\circ} \mathrm{C}$. 降解菌基因组的提取参照文 献 [9], 采用对大多数细菌和古生菌 16S rDNA 基因 V3-V5 区具有特异性的引物对 341F(5'-CCTACGGGAGGCAGCAG)和907R(5'-CCGTCAATTCA/CTTTGAGTTT)对提取的样品基因组进 行 PCR 扩增, 其中引物 341F 的 5’端连接着 40 个碱基的 GC 发夹结构 (5'-CGCCCGCCGCGCG CGGCGGGCGGGGCGGGGGCACGGGGGC). 采用Touchdown反应策略, 条件如下: $95^{\circ} \mathrm{C}$ 预变性 $5 \mathrm{~min}$, 以 下为 $94^{\circ} \mathrm{C} 1 \mathrm{~min}, 65-56^{\circ} \mathrm{C} 30 \mathrm{~s}$ (每个循环降 $1^{\circ} \mathrm{C}$, 共 10 个循环), $72^{\circ} \mathrm{C} 1 \mathrm{~min}$, 后 20 个循环用 $55^{\circ} \mathrm{C}$ 退火, 最后 $72^{\circ} \mathrm{C}$ 下延伸 $30 \mathrm{~min}^{[10]}$.

变性梯度凝胶电泳(DGGE) 条件为：聚丙烯酰胺凝胶浓度 $6 \%$, 变性梯度 $40 \%-70 \%(100 \%$ 变性剂为 $7 \mathrm{~mol} / \mathrm{L}$ 尿素和 $40 \%$ 去离子甲酰胺的混合物), 电泳缓冲液为 $1 \times \mathrm{TAE}, 60^{\circ} \mathrm{C}$ 条件下 $100 \mathrm{~V}$ 电泳 $16-18 \mathrm{~h}$. 聚丙烯 酰胺凝胶用SYBR Green I(用TAE稀释10000倍)染色 $30 \mathrm{~min}$ 后, 用Omega $10^{\mathrm{TM}}$ 全自动多功能凝胶成像分析 系统拍照.

\section{5 降解菌的系统发育分析}

从聚丙烯酰胺凝胶上切下条带, 放到 $1.5 \mathrm{ml}$ 离心管中捣碎, 加人 TE缓冲液 $30 \mu \mathrm{l}, 4^{\circ} \mathrm{C}$ 过夜使DNA溶出. 取离心后上清为模板, 用上述同样的引物(其中 $341 \mathrm{~F}$ 不含 GC夹)和程序扩增, PCR产物送上海生工生物工 程技术有限公司测序 ${ }^{[11]}$. 将测得的 $16 \mathrm{~S}$ rDNA序列在GenBank数据库中进行相似性比较, 选取同源性较高 的相关菌株16S rDNA序列作为参比对象, 用Clustal W进行多重序列比对, 然后采用MEGA 4.0软件中的 邻接法构建系统发育树 ${ }^{[12]}$. 测序结果同时提交到GenBank获得序列号为EU887518到EU887526.

\section{2 实验结果}

\section{1 化学组成分析}

MEPS含鼠李糖、阿拉伯糖、甘露糖、葡萄糖和半乳糖5种中性单糖, 摩尔比为7.4:6.6:1.4:1.0:1.0. 含 葡萄糖醛酸和半乳糖醛酸两种酸性单糖, 糖醛酸含量为 $6.7 \%$, 蛋白质含量为 $8.3 \%$, 等离子光谱分析结果 显示, MEPS含多种金属元素, 其中Ca和Mg含量较高(表1).

表 1 MEPS 金属元素含量 $(\mathrm{mg} / \mathrm{g})$

Tab.1 Metal content of MEPS

\begin{tabular}{lllllllllll}
\hline 金属元素 & $\mathrm{Al}$ & $\mathrm{Ba}$ & $\mathrm{Ca}$ & $\mathrm{Cu}$ & $\mathrm{Fe}$ & $\mathrm{Mg}$ & $\mathrm{Mn}$ & $\mathrm{Na}$ & $\mathrm{Sr}$ & $\mathrm{Zn}$ \\
\hline 元素含量 & 0.130 & 0.023 & 44.142 & 0.144 & 0.098 & 10.037 & 0.059 & 0.678 & 0.262 & 0.114 \\
\hline
\end{tabular}

\subsection{MEPS 降解过程分析}

将稳定的富集培养菌液(来自于 2007 年 8 月太湖水华期水样)接种到含 MEPS 培养基中后, 发现MEPS 在最初的几天里降解较快(图 1), 大约 $18 \mathrm{~d}$ 后, 即碳水化合物减少约 $60 \%$ 后, 降解过程放慢, 到 $37 \mathrm{~d}$ 即实 验结束时仍有约 $30 \%$ 的碳水化合物未被微生物利用. 伴随着多糖的降解, 细菌数量明显增长, 在大约 $18 \mathrm{~d}$ 时数量达到最大约 $8.0 \times 10^{8} \mathrm{cells} / \mathrm{ml}, 27 \mathrm{~d}$ 后细菌数量开始缓慢下降. 2008 年 5 月采自太湖的水华期微生物 水样同样显示了对 MEPS 的降解能力(数据未显示). 而来自于太湖无水华期的微生物水样接种到含 
MEPS 的培养基后, 转接 3 次仍得不到稳定的 MEPS 降解菌群, 培养至 37d 后碳水化合物减少量最多也只 有 $5 \%$ 左右. 接种浦口无水华水体中的微生物水样并 3 次转接进行富集培养, 均未发现碳水化合物的减少.

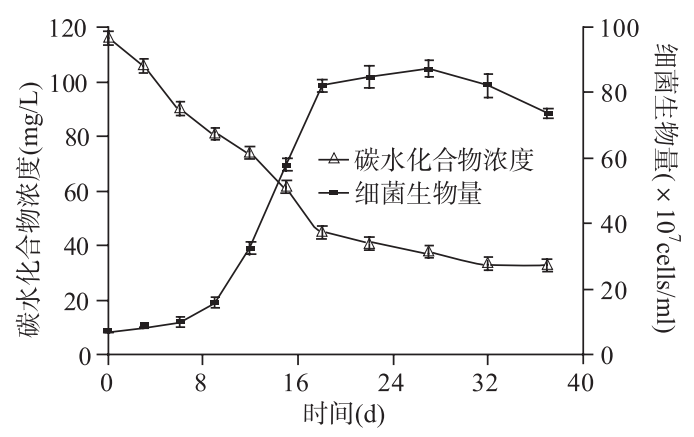

图 1 铜绿微囊藻胞外多糖降解过程中碳水化合物浓度和细菌生物量的变化

Fig.1 Carbohydrate concentration and bacterial growth during breakdown of the exopolysaccharide from

$$
\text { M. aeruginosa FACHB-912 }
$$

\subsection{PCR-DGGE 分析降解菌组成}

取不同降解时期的菌样做 DGGE 分析, 共得到了大约 9 个条带(图 2), 从 DGGE 图中看出整个降解过 程中条带组成基本不变, 也就是说降解菌的组成基本不变，只是在 $18 \mathrm{~d}$ 后，一些之前很浅的条带开始变 得清晰一些.

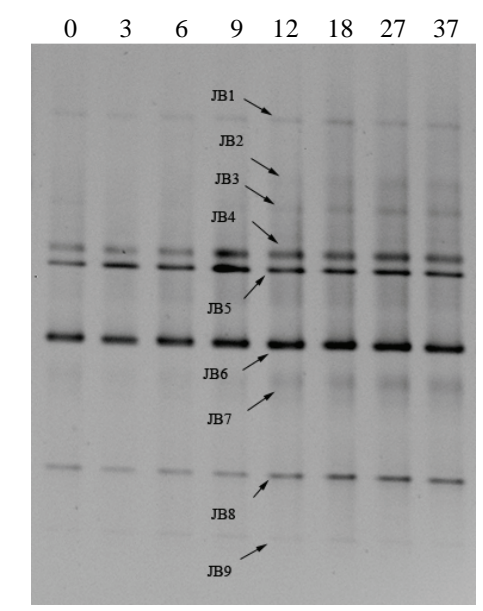

图 2 铜绿微囊藻胞外多糖降解过程中细菌组成 (顶部数字为培养天数, “个”标记为割胶回收的 9 个条带)

Fig.2 Composition of the microbial community during breakdown of the exopolysaccharide from M. aeruginosa FACHB-912

\section{4 降解菌的系统发育分析}

从聚丙烯酰胺凝胶上切下 9 条带, 再次 PCR 后进行测序. 从系统发育树(图 3)来看, 条带 JB4 代表的 菌属于 $\alpha$-变形菌亚纲(Alpha proteobacteria), 条带 JB1、JB2 和 JB5 代表的菌属于鞘氨醇单胞菌属 (Sphingomonas), 条带 JB7 代表的菌属于褐螺菌属(Phaeospirillum), 条带 JB3 代表的菌属于假单胞菌属 (Pseudomonas), 条带 JB8 代表的菌属于红环菌科 (Rhodocyclaceae), 条带 JB6 代表的菌属于 Hylemonella(无译名), 条带 JB9 代表的菌属于放线菌纲(Actinobacteria)中的分枝杆菌属(Mycobacterium). 


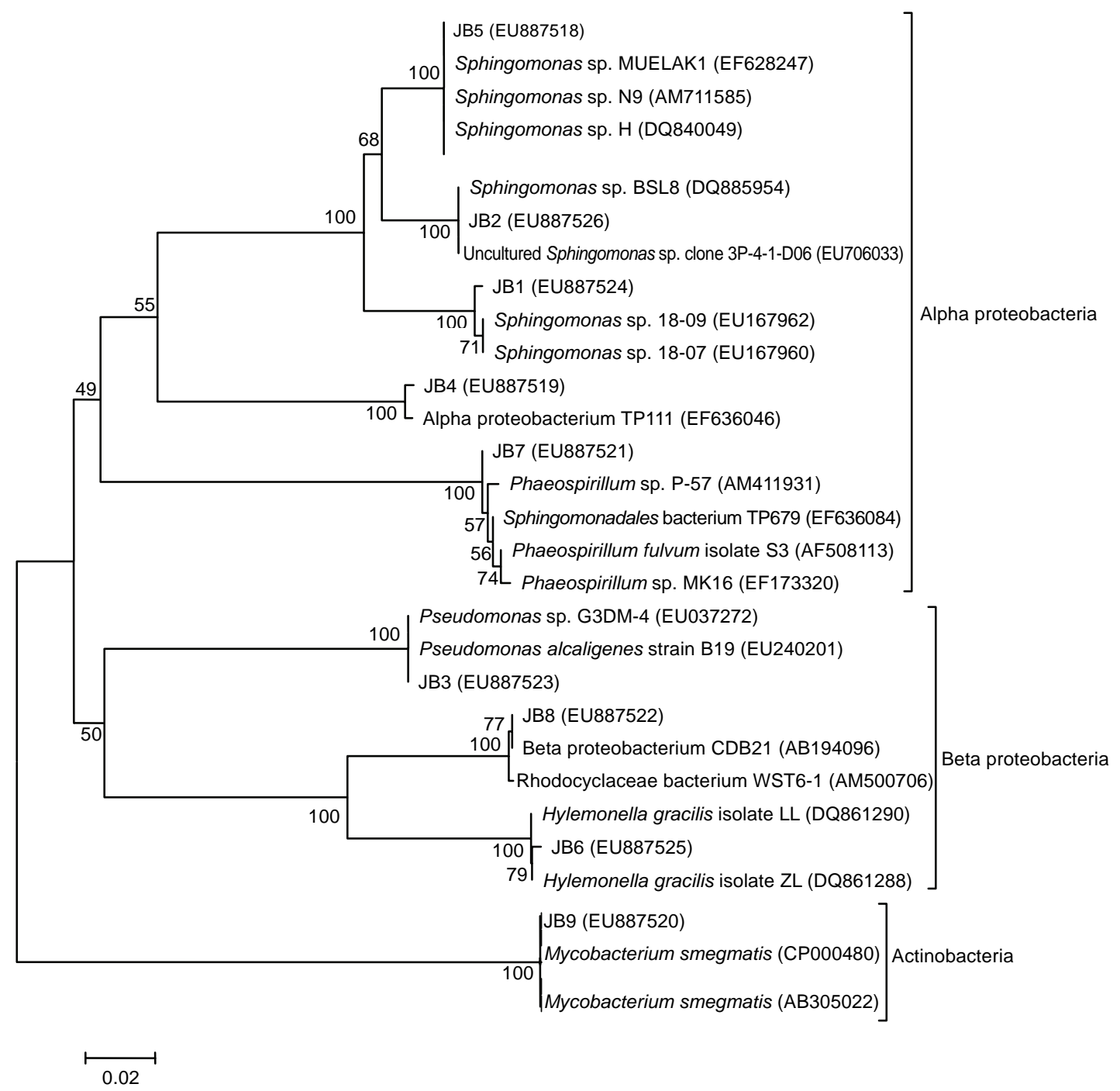

图 3 铜绿微囊藻胞外多糖降解菌群的系统发育树

(括号内为序列的登录号; 每个分支点上的数字为引导值的支持百分率;

刻度尺表示每个核苷酸位置的替换率为 $2 \%$ )

Fig.3 Phylogenetic tree of bacteria degrading the exopolysaccharide of M. aeruginosa FACHB-912

\section{3 讨论}

从铜绿微囊藻中分离的胞外多糖其化学组成与已报道的微囊藻胞外多糖化学组成类似, 都是酸性杂 多糖 ${ }^{[13-14]}$.

从MEPS的降解过程看(图1), 经过37d的培养大部分MEPS被降解利用, 剩下约 $30 \%$ 的碳水化合物应 该是未能被降解的多糖或者寡糖片段, 这可能是因为MEPS结构复杂, 有些成分的降解需要一个更长期 的过程, 或者需要这个菌群以外的细菌参与, 这说明在自然界中的微囊藻胞外多糖是可以被微生物降解 的. 接种采自2007年和2008年太湖水华期的微生物水样后, MEPS都能被显著降解, 说明水华暴发期的水 体菌群能够有效地降解MEPS. 而接种来自于太湖无水华期以及夏季无水华水体中的微生物水样, MEPS 
都没有被显著降解, 这表明在没有水华的水体中可能没有稳定的能够降解MEPS的微生物菌群的存在. 由 于MEPS结构复杂, 它的降解需要特殊的降解酶, 只有能产生这些酶的细菌才能有效降解MEPS, 而这类菌 群存在的前提是环境中存在大量的MEPS, 当环境中没有MEPS时, MEPS降解菌群就缺失, 因此, 稳定的 MEPS的降解菌群只存在于微囊藻水华暴发的阶段. Janse等研究了赤潮种类棕囊藻(Phaeocystis)胞外多糖的 微生物降解过程, 30d后降解速度显著减慢, 经过了 $48 \mathrm{~d}$ 仍有约 $10 \%$ 的碳水化合物未能被降解利用 ${ }^{[15]}$. Giroldoi等研究了一种鼓藻(Staurastrum orbiculare)胞外多糖的微生物降解过程, 该多糖在14d后就基本完 全降解 ${ }^{[16]}$. 这显示自然界中不同藻类多糖的微生物降解过程和速度可能是不同的.

从富集培养菌液中没有能分离到具有降解MEPS能力的单个菌株, 暗示了MEPS的降解可能是降解菌 群共同作用的结果. 系统发育分析显示这些降解菌分属于不同的类群, 表明MEPS的降解是多种类群细 菌参与完成的, 由于MEPS含有蛋白质, 降解菌群中的部分细菌可能依靠蛋白质生长, 部分细菌可能是依 靠MEPS的降解产物生长, 但至少其中一些细菌能够直接降解MEPS.

\section{4 参考文献}

[1] Plude JL, Parker DL, Schommer OJ et al. Chemical characterization of polysaccharide from the slime layer of the cyanobacterium Microcystis flos-aquae C3-40. Applied and Environmental Microbiology, 1991, 57: 1696-1700.

[2] Parker DL, Schram BR, Plude JL et al. Effect of metal cations on the viscosity of a pectin-like capsular polysaccharide from the cyanobacterium Microcystis flos-aquae C3-40. Applied and Environmental Microbiology, 1996, 62: 1208-1213.

[3] Tien CJ, Krivtsov V, Levado E et al. Occurrence of cell-associated mucilage and soluble extracellular polysaccharides in Rostherne Mere and their possible significance. Hydrobiologia, 2002, 485: 245-252.

[4] Naeem S, Hahn DR, Schuurman G. Producer-decomposer co-dependency influences biodiversity effects. Nature, 2000, 403: $762-764$.

[5] 李铁林, 吴昌贤, 张燕霞. 糖和糖醇的气相色谱分析研究 II. 糖腈乙酰酯衍生物气相色谱分析的改进. 分析化学, 1982, 10(5): 272-276.

[6] John T, Galambos. The reaction of carbazole with carbohydrates, 1. Effect of borate and sulfamate on the carbozole color of sugars. Analytical Biochemistry, 1967, 19: 119-132.

[7] Liu D, Wong PTS, Dutka BJ. Determination of carbohydrate in lake sediment by a modified phenol-sulfuric acid method. Water Research, 1973, 7: 741-746.

[8] Porter KG, Feig YS. The use of DAPI for identifying and counting aquatic microflora. Limnology and Oceanography, 1980, 25: 943-948.

[9] Boström KH, Simu K, Hagström Å et al. Optimization of DNA extraction for quantitative marine bacterioplankton community analysis. Limnology and Oceanography: Methods, 2004, 2: 365-373.

[10] Kousuke I, Manabu F. Optimization of annealing temperature to reduce bias caused by a primer mismatch in multitemplate PCR. Applied and Environmental Microbiology, 2004, 67: 3753-3755.

[11] 赵兴青, 杨柳燕, 陈 灿等. PCR-DGGE技术用于湖泊沉积物中微生物群落结构多样性研究. 生态学报, 2006, 26(11): 3610-3616.

[12] Saitou N, Nei M. The neighbour-joining method: a new method for reconstructing phylogenetic trees. Molecular Biology and Evolution, 1987, 4: 406-425.

[13] 梅秋红, 缪月秋, 张成武等. 铜绿微囊藻(Microcystis aeruginosa var.m ajor)胞外酸性多糖的分离、纯化及其理化特性. 湖 泊科学, 2005, 17(4): 322-326.

[14] 王习达, 吴国荣, 陈景耀等. 铜绿微囊藻酸性多糖的分离、纯化与结构研究. 中药材, 2003, 26(12): 865-867.

[15] Janse I, van Rijsselm, Ottema A et al. Microbial breakdown of Phaeocystis mucopolysaccharides. Limnology and Oceanography, 1999, 44: 1447-1457.

[16] Giroldo D, Vieira AAH, Paulsen BS. Microbial degradation of extracellular polysaccharides released by a tropical strain of Staurastrum orbiculare (Zygnematophyceae). Phycologia, 2005, 44: 671-677. 